\section{Factors Contributing to the Success of NIH-Designated Underrepresented Minorities in Academic and Nonacademic Research Positions}

\author{
Luis R. Martinez, ${ }^{\dagger}$ Dwayne W. Boucaud, ${ }^{\ddagger}$ Arturo Casadevall, ${ }^{\S}$ and Avery August ${ }^{\| *}$ \\ 'Department of Biological Sciences, Border Biomedical Research Center, University of Texas at \\ El Paso, El Paso, TX 79968; ‘Department of Biomedical Sciences, Quinnipiac University, Hamden, \\ CT 06518; " Department of Molecular Microbiology and Immunology, Johns Hopkins Bloomberg \\ School of Public Health, Baltimore, MD 21205; "Department of Microbiology and Immunology, \\ Cornell University, Ithaca, NY 14853
}

\begin{abstract}
We report the outcomes of a survey of underrepresented minorities (URMs) in life science academic (e.g., faculty) and nonacademic (e.g., research-related) positions seeking to ascertain variables that contribute to their success (e.g., favorable or desired outcome). Given that they had positions in research careers, all respondents were presumed to be successful, and we sought to identify shared factors that were associated with this success. As in previous studies, respondents reported that undergraduate research opportunities, performing research in small- to medium-sized laboratories, and access to mentors throughout all stages of training were important factors for success in their careers. Surprisingly, analysis of the survey results suggests that a record of publications in high-impact factor journals was not essential for their success. There were fundamental differences in the experiences and needs of URMs in academic and nonacademic careers. For example, academic URMs ranked having mentorship as their first choice in order of importance compared with the nonacademic respondents, who ranked this category as their fifth selection. These findings suggest that taking diverse approaches toward these groups is critical for ensuring that all of the most creative minds have an equal opportunity to contribute to realizing our national research goals and diversified workforce.
\end{abstract}

\section{INTRODUCTION}

As the general population in the United States becomes more heterogeneous, the need for increased diversity in the academic and research pipeline is increasingly apparent (Gibbs et al., 2016). The job market in the natural sciences has become highly competitive for those wishing to secure positions as faculty and researchers. Scientists from the traditionally defined minority groups (National Institutes of Health [NIH]-designated underrepresented minority [URM] groups; e.g., African Americans, Hispanic/Latino, and Native Americans) continue to be underrepresented relative to their numbers in the U.S. population. A possible major factor in perpetuating these disparities is the lack of faculty diversity in the sciences (Hassouneh et al., 2014; Griffin et al., 2015). Despite efforts to recruit and retain URM faculty in the last three decades, particularly in the life and physical sciences, only modest improvements have been achieved (Allen-Ramdial and Campbell, 2014). As a result, many capable and talented students have opted out of pursuing scientific careers despite the investment of a significant amount of time, resources, and efforts by a number of governmental and nongovernmental organizations who hope to increase diversity in this area (Gibbs et al., 2014). Additionally, there are concerns that shortage of URM faculty directly affects recruitment, mentoring, and retention of minority students, given
Kathryn E. Perez, Monitoring Editor Submitted Sep 29, 2016; Revised Mar 20, 2018; Accepted Mar 23, 2018

CBE Life Sci Educ June 1, 2018 17:ar35

DOI:10.1187/cbe.16-09-0287

Conflicts of interest: L.R.M., D.W.B., and A.C. are members of the American Society for Microbiology's Committee on Microbiological Issues Impacting Minorities. L.R.M. serves as a Leshner Leadership Institute Public Engagement Fellow in infectious diseases with the American Association for the Advancement of Science. A.C. is a member of the American Association of Immunologists Minority Affairs Committee. *Address correspondence to: Avery August (averyaugust@acornell.edu).

() 2018 L. R. Martinez et al. CBE-Life Sciences Education @ 2018 The American Society for Cell Biology. This article is distributed by The American Society for Cell Biology under license from the author(s). It is available to the public under an Attribution-Noncommercial-Share Alike 3.0 Unported Creative Commons License (http://creativecommons.org/licenses/ by-nc-sa/3.0)

"ASCB $®$ " and "The American Society for Cell Biology ${ }^{\circledR}$ " are registered trademarks of The American Society for Cell Biology. 
that they serve as important role models and mentors to minority trainees and promote academic excellence that enhances students' outcomes (Price et al., 2005; Leboy and Madden, 2012; Rodriguez et al., 2014).

In 2012, the American Institute for Research and the Institute for Higher Education Policy called for action to broaden participation and increase diversity in the science, technology, engineering, and mathematics (STEM) disciplines (Rodriguez et al., 2012). They found that the definition of URM success was too narrow and proposed an expansion of the concept to much more than an individual merely obtaining a higher education degree. It was established that the status quo of our education system and the distribution of federal funding, mostly concentrated in well-resourced 4-year institutions, is preparing URM students to be first-line bench-science employees. A lack of leadership training diminishes their preparation to develop adequately to become future leaders, particularly in the sciences. Federal funding agencies such as the NIH and National Science Foundation (NSF) have recently created initiatives to identify social and psychological factors that mitigate individual and institutional barriers to workforce diversity in order to develop a scalable strategy to effectively disseminate and sustain diversity within the nationwide scientific workforce. For instance, the INCLUDES (Inclusion across the Nation of Communities of Learners of Underrepresented Discoverers in Engineering and Science) initiative recently launched by the NSF aims to help researchers develop innovative, scalable strategies to make STEM broadly inclusive. Similarly, current events related to issues of diversity in higher education, such as the resignation of the University of Missouri's president (Peralta, 2015), the Fisher vs. the University of Texas case on affirmative action (Espinosa and McDonough, 2016), Yale University's public struggles in diversifying their faculty (McMurtrie, 2016), and many others, highlight the importance of the commitment on the part of the U.S. government and higher education institutions to provide a more inclusive research workforce and learning environment.

Many studies have focused on describing the factors affecting recruitment and retention of URM faculty in higher education, suggesting that barriers to opportunities and participation continue to limit success (Fang et al., 2000; Leboy and Madden, 2012; Whittaker and Montgomery, 2012; Whittaker et al., 2015). For instance, poor or absent mentorship, unclear criteria for tenure and promotion, and misunderstanding of the institutional culture have been described as being among the most common challenges in the recruitment and retention of URMs (Mahoney et al., 2008; Pololi et al., 2010; Whittaker et al., 2015). Other reports suggest that academic institutions have been more effective at recruiting URM faculty and less successful at helping them to attain tenure and promotion (Fang et al., 2000; Leboy and Madden, 2012). Recent evidence indicates that URM faculty are less likely to be promoted (Fang et al., 2000) and are likely to receive less research grant support than non-URM colleagues (Ginther et al., 2011). Similarly, a cultural perception that an institution is not supportive for URM faculty advancement diminishes individuals' morale and increases the likelihood that such faculty will leave academia prematurely (Price et al., 2005; Cropsey et al., 2008; Zambrana et al., 2015).

Other studies have focused on the reasons that URM life scientists abandon academic and research careers (Palepu et al., 2000; Cropsey et al., 2008; Rodriguez et al., 2014). To effectively address and stop the brain drain from the research pipeline (Allen-Ramdial and Campbell, 2014), it is important to understand the reasons for which URM scientists in the life sciences leave academia. However, it is equally important to identify factors that are responsible for the success of URM life scientists, in both academic and nonacademic environments, to provide empirically driven solutions and opportunities for potential policy and other changes. Currently, few or no data are available on factors correlating with the success of URM faculty. Searches of PubMed, Scopus, and Science Direct databases for studies that shed light on this problem (using combinations of the following keywords: success, underrepresented minority, faculty, STEM, life sciences, and medicine) failed to reveal any relevant studies. To address the paucity of research on success factors for URM scientists and understand the needs of this population in the workforce, we therefore developed a voluntary survey of successful URM scientists in diverse areas of the life sciences, which covered career-related aspects in each stage of their individual paths to success in being recruited and promoted in their careers, including faculty in higher education. We considered that respondents were successful, because they were in research careers, and aimed to determine factors associated with this success that may lead to the development of more effective strategies for increasing and retaining academic (faculty) and nonacademic (researchers or others in research-related positions) URM in the life sciences. It is essential to note that only URM scientists were invited to participate in the study, as we exclusively wanted to document their perspectives and experiences about their success in the life sciences, which may or may not be similar in other groups. Although it is important to compare URMs versus non-URMs in the future, this was outside of the scope of the present study.

\section{METHODS}

\section{Survey Demographics and Dissemination}

The survey population consisted of life scientists self-identifying as belonging to one or more of the NIH-designated URM groups: African American, Hispanic/Latino, or Native American. The participants were invited to complete the survey via a link by email, which was disseminated primarily via the minority affairs committees (MACs) or equivalents of the following professional societies: the American Association of Immunologists (AAI), the American Physiological Society, the American Society for Biochemistry and Molecular Biology, the American Society for Cell Biology, the American Society for Microbiology (ASM), the Biochemical Engineering Society, the Endocrine Society, the Society for Neuroscience, and the Society for the Study of Reproduction. In some cases, email requests to participate were sent directly to identified URM faculty. The survey was shared and discussed with the MACs of the indicated societies and modified based on their feedback to enhance clarity, acceptability, and feasibility. In addition, the ASM's Committee on Microbiological Issues Impacting Minorities (CMIIM) validated the contents of the survey before it was widely disseminated. The invitation email described the objective of the study and how the data would be collected and disseminated and stated that the participation in the survey was voluntary (see the Supplemental Material for a copy of the invitation email). 
Individuals received access to the survey if they agreed to participate on the consent form.

Although this is a convenience sample in the sense that was accessible to study, we believe that the sampled population is likely to represent the broader URM population, given that these are diverse disciplines that include broad swaths of the biological sciences (Supplemental Figure 1). These nine societies are likely to include a significant proportion of URMs in the biological sciences.

\section{Survey Design}

The survey consisted of 41 questions (see the Supplemental Materials for survey questions). The survey collected data on the following:

1. Career: We assumed that success can be partially defined as the length a scientist stays in a position. Therefore, we asked respondents about their primary scientific disciplines and the number of years in their career posts and their current institutions at the time of the survey.

2. Education and training: We wanted to learn about the level of education and training of academic and nonacademic respondents to understand how their backgrounds influenced their success. We asked questions about the type (e.g., majority vs. minority serving) and size of institutions in which they obtained their degrees; early research exposure; transition preparation between undergraduate, graduate, and postdoctoral (if any) training and professional careers; laboratory sizes; and time in each training level.

3. Publication record: We investigated whether the respondents considered high-impact factor publications important for their success. Each respondent was asked to self-report whether they have or have not published in journals with low (IF $<3$ ), medium (IF $>3$ ), or high (IF $>7$ ) impact factors.

4. Professional membership: We assessed the role that professional societies play in the success of URMs. Thus, respondents were asked whether or not they were affiliated with a professional organization, time, involvement (e.g., phase of training or URM committee), number of URM population, and importance of these societies and their activities in success.

5. Family and non-science role models: Another important aspect in the success of URMs is the support system surrounding these individuals, which include family and other non-science role models. Respondents were surveyed on the education level at home, role model relationship with them, whether or not family members were scientists, and training phases in which a role model was identified.

6. Academic versus nonacademic positions: Respondents were asked about their positions and separated into academic and nonacademic researchers. Data were collected regarding the types and sizes of their current institutions and whether or not there were significant URM populations in their organizations.

These topics, including the possible enablers of success provided, were chosen based on the personal experiences of the CMIIM members and the authors, who are URMs with positions in academic and nonacademic (e.g., government or private sectors) settings. To ensure confidentiality, data from the survey were collected anonymously, and participants were not identified. The aggregated responses were analyzed. This survey was conducted following approval by the Institutional Review Board of Cornell University, Ithaca, NY.

\section{Statistical Analysis}

Data were analyzed using SPSS statistics version 23.0 (IBM, Armonk, NY). Descriptive and frequency responses for each question were compiled and calculated, respectively. Furthermore, participants' comments to open-ended questions were also gathered and coded for analysis. Two sets of analyses were performed. In the first, tests of association were performed using the Pearson's chi-square test (exact significance twosided, where computationally possible), with Bonferroni correction for number of tests. When assumptions of the chi-square tests were not met, a Fisher's exact test was performed and, correcting for the Bonferroni adjustment, $p$ values of $<0.0015$ were considered significant. In the second analysis, binary responses were also analyzed with generalized linear models, using binary logistic to obtain odds ratios (with Bonferroni correction for pairwise comparisons), to determine the association of the responses (Supplemental Tables 1 and 2). In those cases, the within-group multiple-comparison and Bonferroni corrections were automatically performed by SPSS, such that $p$ values of $<0.05$ were considered significant.

\section{RESULTS}

\section{Demographics}

On the basis of the membership of URMs in the societies with the most respondents (e.g., AAI and ASM) and the number of responses received, we estimate that the response rate was $\sim 23 \%$. Three hundred and twelve URM life scientists responded to the survey, and they self-identified as African American (41\%), Hispanic/Latino (50\%), Native American (5\%), or other (4\%; Supplemental Figure 1). The scientific disciplines represented by the respondents included: immunology (40\%), microbiology (13\%), biochemistry (7\%), cell biology $(7 \%)$, molecular biology (6\%), neuroscience $(4 \%)$, physiology $(3 \%)$, and several others (20\%; Supplemental Figure 1). Sixty-six percent of the respondents were faculty members at an academic institution (including the NIH), whereas the other 34\% worked at nonacademic institutions, including government or private sector institutions (Supplemental Figure 1). The majority of the respondents had been in their posttraining career for 1-10 years (60\%; 39\%, $1-5$ years; $21 \%, 6-10$ years) and also at their current institution for $1-10$ years (70\%; $51 \%, 1-5$ years; $19 \%$, 6-10 years; Supplemental Figure 2) at the time of the survey. Fifty-seven percent indicated that there was low URM presence at their current institutions, with $68 \%$ of them working at either small $(18 \% ;<10,000$ students) or large (50\%; >10,000 students) majority-serving institutions and $22 \%$ working at either small ( $12 \% ;<7,000$ students) or large $(10 \% ;>7,000$ students) minority-serving institutions. Only $9 \%$ of the respondents attended community college (Supplemental Figure 2). Sixty-seven percent of the respondents obtained their undergraduate degrees from small (19\%) or large (48\%) majority-serving institutions compared with 33\% who attended either small (19\%) or large (14\%) minority-serving institutions. In this regard, $72 \%$ of the respondents obtained their $\mathrm{PhD}$ degrees from large majority-serving institutions. Most of the respondents $(78 \%)$ worked on their $\mathrm{PhD}$ thesis research in laboratory 
groups of small (1-5 members; 38\%) or medium (6-10 members; 40\%) size. A similar tendency was observed in respondents who pursued postdoctoral training (80\%). Interestingly, $63 \%, 31 \%$, and $8 \%$ of these individuals reported having had one, two, and three different postdoctoral training appointments, respectively, with the majority of those fellowships lasting 1-3 years. We used generalized linear models to determine potential associations between career-related questions and questions in other areas that were considered important for success. We considered the following factors: education and training, publication record, membership in professional societies, and family and non-science role models.

\section{Education and Training}

We analyzed responses related to education and training to identify potentially shared features of the survey participants, who were all considered to be successful. At the undergraduate level, there was a significant difference in the time respondents had been in their careers and for those who began their undergraduate degrees at a community college, $\chi^{2}(4)=9.494$, $p<0.05$. Those who had been $5-10$ years in their careers were more likely to have begun their undergraduate degrees at a community college compared with those who had been in their careers for 20 years or more (odds ratio [OR] $=5.851, p$ $<0.03)$. These findings suggest that community college attendance, while proportionally low among participants, is a larger factor for those who are recently starting their careers compared with those with established careers. There was also a significant difference in respondent participation in undergraduate research and time in career, $\chi^{2}(4)=15.765, p<$ 0.003 . Pairwise comparison further revealed a strong association between respondents with 5-10 years in their careers and their participation in undergraduate research compared with those who had been in their careers for 20 years or more (OR $=8.207, p<0.014$, with Bonferroni correction for multiple comparisons). Participants who had performed undergraduate research were more likely to have felt that they were prepared for the transition from undergraduate to graduate school compared with those who felt they were not prepared or unsure whether they were prepared for this transition (OR $=2.38, p<0.006$, with Bonferroni correction for multiple comparisons), and less likely to have attended small minority-serving institutions compared with those who attended large majority-serving institutions for their undergraduate degrees $(\mathrm{OR}=0.453, p<0.05)$. Moreover, this group was more likely to get postdoctoral training in larger labs ( $>16$ persons) compared with those who did not participate in undergraduate research ( $>20$ persons, $\mathrm{OR}=6.389, p<0.05 ; 16-20$ persons, $\mathrm{OR}=11.111, p<0.04)$. Those who felt they were prepared for the transition from undergraduate to graduate school were more likely to currently be in academic positions than those who felt they were not prepared for this transition (OR $=2.202, p<0.005$, with Bonferroni correction for multiple comparisons). Participation in undergraduate research, which is more likely to happen at a large majority-serving institution, was therefore associated with being prepared for transition to graduate school and was likely to be followed by postdoctoral training in a larger ( $>16$ persons) lab. Furthermore, respondents in academic positions were more likely to report feeling prepared for transition to graduate school.

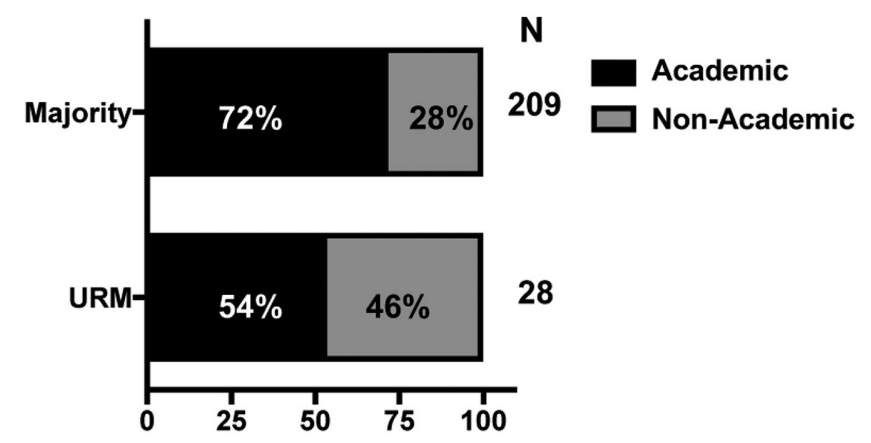

FIGURE 1. Respondents who received PhDs from majority institutions are more likely to be in academic positions. Bars indicate percentage of respondents currently in academic or nonacademic positions vs. where they received their PhDs (majority- or URM-serving institutions). $N$ indicates number of responses for each category of those who received PhDs from a majority or predominantly URM institution.

At the graduate level, analysis of academic positions revealed a significant difference in the majority- versus minority-serving institutions where respondents received their $\mathrm{PhDs}$ (regardless of size), $\chi^{2}(1)=3.63, p<0.05$, and those receiving PhDs from minority-serving institutions (regardless of size) were less likely to be in an academic position compared with those receiving $\mathrm{PhDs}$ from majority-serving institutions $(\mathrm{OR}=0.454, p<0.05)$. This suggests that respondents who received $\mathrm{PhDs}$ at minority-serving institutions were less likely to have academic positions than those who received $\mathrm{PhDs}$ from majority-serving institutions (Figure 1).

\section{Publication Record}

We analyzed responses related to respondents' publication records to identify potentially shared features of the survey participants. They were asked to self-report their publication records and the impact factors (IF; e.g., lower IF, $<3$; medium IF, $>3$; higher IF, $>7$ ) of the journals in which they published at different stages of their training (Supplemental Figure 3). The majority of the participants did not publish during high school (99\%) or college $(67 \%)$. For the $33 \%$ of the participants who published articles as undergraduate students, the majority were published in lower-IF (59\%) or medium-IF (34\%) journals. During graduate school, the majority of the respondents (93\%) reported that they had published the results of their thesis research mostly in journals with medium (58\%) and higher (29\%) IFs. Although the IFs of journals in which participants published during postdoctoral training increased (medium IF, 38\%; higher IF, 55\%) compared with IFs of journals in which they published in graduate school (medium IF, 32\%; higher IF, $26 \%$ ), there was also an increase in the number of participants (postdoctoral, 13\%, vs. graduate, 7\%) who did not publish an article during the postdoctoral period of training (Supplemental Figure 4). There was a significant association between having a current position in an academic institution and publications during training, $\chi^{2}(3)=18.376, p<0.0001$. Pairwise comparisons revealed that those who published as postdocs were more likely to be in academic positions than those who published as graduate students (OR $=4.545, p<0.004$, with Bonferroni correction for multiple comparisons). Analysis of the 


\section{Academic or non-academic affiliation and publications}

A)
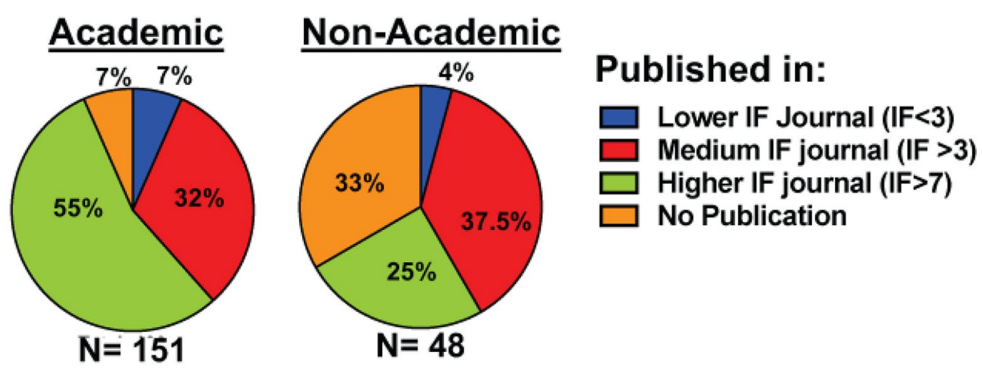

B)
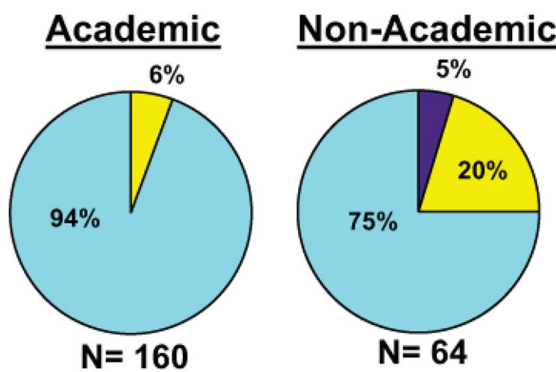

FIGURE 2. Impact factor (IF) of journals in which respondents published is less important than publishing as postdocs for those in academic positions. (A) IF of journal in which respondents published as postdocs with whether they were currently faculty members at an academic institution (including the NIH). (B) Publishing (regardless of journal IF) as undergraduates, graduate students, or postdocs with whether they were currently faculty members at an academic institution (including the NIH). $N$ indicates number of responses.

IFs of those publications revealed no significant difference across IF of publications and current position at an academic institution. Notably, while there was a significant association in the publication record as a postdoc and current position at an academic institution, $\chi^{2}(3)=25.005, p<0.0001$, this was only between those who published as postdocs and those who did not, regardless of the IF of the publication (lower IF vs. no publication $\mathrm{OR}=8, p<0.02$; medium IF vs. no publication $\mathrm{OR}=$ 4.267, $p<0.003$; high IF vs. no publication $\mathrm{OR}=11.067$, $p<0.0001)$. This suggests that, for those who currently have academic positions, publishing papers during the postdoctoral period was more important than the IFs of the journals in which they were published (Figure 2).

Publications during specific training periods (undergraduate, graduate, or postdoc) were not associated overall with the type of institution where participants received their $\mathrm{PhDs}$ (majority vs. minority serving), $\chi^{2}(2)=5.227, p=0.073$. Those who felt they were prepared for the transition from undergraduate to graduate school were more likely to have published papers in medium-IF (OR $=4.211, p<0.01$ ) or high-IF (OR $=3.364, p<0.05)$ journals as graduate students compared with those who felt they were not prepared. Among those who felt they were prepared for the transition from undergraduate to graduate school compared with those who felt they were not prepared, there was also a significant difference in those who published as undergraduates versus as graduate students $(p<0.0001)$ or as postdocs $(p<0.0001)$. Thus, feeling prepared while transitioning between undergraduate and graduate school was associated with subsequent publication of papers in higher-IF journals.

\section{Professional Membership}

We analyzed responses related to respondents' membership in professional societies to identify potentially shared features of the survey participants. URM scientists were surveyed about their involvement with professional scientific organizations and the role of those organizations in their career success, because URM participation in scientific societies has been suggested to be a factor leading to retention and success (Chemers et al., 2011; Supplemental Figure 5). Ninety-eight percent of the URM scientists who responded to the assessment are members of a professional scientific organization, which is likely related to the manner of dissemination of the survey. Only $25 \%$ of the participants became affiliated with a professional society as undergraduate students, whereas $73 \%$ were members of a professional organization during their graduate training. Fifty-seven percent of those affiliated reported that there were not a significant number of URM members in the professional societies in which they were most active.

There was a significant association between the time period when participants were members of professional societies and whether they were in academic positions, $\chi^{2}(2)=82.641, p<0.0001$. Respondents who were members as graduate students $(\mathrm{OR}=0.022$, $p<0.0001$, with Bonferroni correction for multiple comparisons) or postdocs (OR $=0.061, p<0.0001$, with Bonferroni correction for multiple comparisons) were less likely to be in academic positions compared with those who were members as faculty. Furthermore, while there was no association between current membership in a professional society and years in career, there was a significant association between those who had obtained postdoctoral training and current membership in a professional society, $\chi^{2}(1)=5.82, p<0.02$, and respondents who had obtained postdocs were more likely to belong to a professional society (OR $=12.977, p<0.03$ ). However, the number of postdocs obtained, the length of those postdocs, or the size of the laboratory groups in which postdocs were done was not significant. This was also not associated with publication records as postdocs. It is probable that academics are more likely to be members of professional societies or to maintain their memberships once they join.

The majority (53\%) of individuals affiliated with a professional society indicated that they are active members of the URM committees in those organizations. Moreover, 76\% indicated that they became active members in a minority committee within their scientific societies after obtaining a faculty/ professional appointment. Only 33 and 28\% participated in URM committees as graduate students and postdoctoral fellows, respectively. Notably, there was a significant difference 
between those who were active members of URM committees in professional organizations and their publication records as postdocs, $\chi^{2}(5)=7.637, p<0.05$. Respondents who did not publish as postdocs were more likely to be active members of URM committees in professional organizations than those who had medium-IF (OR $=2.977, p<0.03)$ or high-IF publications $(\mathrm{OR}=$ $3.509, p<0.01)$ as postdocs. It is possible that URM postdocs who were less productive with regard to publication were more heavily involved in the activities of a professional society as a way of networking and exploring nonacademic careers. Nevertheless, it is also conceivable that URM postdocs involved in committees of a professional society devote too much time to that service activity, thereby affecting their publication productivity.

When asked to rank membership-related elements that we thought might contribute to their career success, many described, in order of preference, that 1) financial support, 2) networking, 3) committee participation, and 4) conferences (e.g., seminars) were the most influential for their career development. Despite this, a remarkable, $68 \%$ of the respondents reported that they do not attribute their professional success to participation in a scientific society.

\section{Family and Non-Science Role Models}

A strong support system is generally considered to be a very important factor for individual success; consequently, we asked participants specific questions about the role of family members and role models inside as well as outside the field of science (Supplemental Figure 6). Participants indicated that the highest degree in their homes during their college educations was less than high school (6\%), high school (26\%), associate's (9\%), bachelor's (20\%), master's (16\%), and doctoral or other terminal degree $(23 \%)$. There was a significant difference between those who had academic positions and the level of education in the home during undergraduate training, $\chi^{2}(5)=13.328$, $p<0.02$. Respondents who as undergraduates lived in homes where the highest degree was an AA degree $(\mathrm{OR}=0.279$, $p<0.02)$ or MS degree (OR $=0.296, p<0.007$ ) were less likely than those who as undergraduates lived in homes where the highest degree was $\mathrm{PhD} / \mathrm{MD}$ or other professional degree to currently have academic positions. Furthermore, respondents who as undergraduates lived in homes where the highest degree was a high school diploma were less likely to participate in undergraduate research compared with those who lived in homes where the highest degree was $\mathrm{PhD} / \mathrm{MD}$ or other professional degree (OR $=0.299, p<0.009$ ), although there was no difference with those who lived in homes where the highest degree was no high school diploma.

Respondents who as undergraduates lived in homes where no one had a high school diploma ( $\mathrm{OR}=0.304, p<0.041$ ) or just a high school diploma (OR $=0.451, p<0.041$ ) were less likely to say that they felt prepared for the transition between undergraduate and graduate school compared with those who lived in homes as undergraduates where a $\mathrm{PhD} / \mathrm{MD}$ or other professional degree was the highest degree.

Only $18 \%$ of the participants had a role model in their family who was a faculty member in higher education. Likewise, only $15 \%$ disclosed having a role model who was a scientist outside higher education. The majority (68\%) indicated having a family member as a role model who was not in any field of science, particularly describing parents and siblings filling that role.
There was a significant association between how respondents felt about their preparation for the transition from undergraduate to graduate school and the presence of a role model in the family who was not in science, $\chi^{2}(1)=5.944, p<0.02$. Surprisingly, respondents who did not have a role model in the family who was in science were more likely to feel that they were prepared for the transition from undergraduate to graduate school $(\mathrm{OR}=2.020, p<0.015)$.

\section{Academic versus Nonacademic Positions}

It is also important to assess whether factors that are associated with success differed between those URMs who pursue an academic versus a nonacademic career. We compared the differences between respondents working in academic versus nonacademic institutions (Supplemental Figure 7). In both groups, most of the respondents came from homes in which a relative had a college degree (e.g., AA, BS, or MS) during the respondents' undergraduate education. There was a significant association between current position in academia and postdoctoral training, $\chi^{2}(1)=14.245, p<0.0001$. Not surprisingly, respondents who obtained postdoctoral training were more likely to be in academic positions compared with those who did not (OR $=3.582, p<0.0001$ ). As mentioned earlier, respondents who received $\mathrm{PhDs}$ at large minority-serving institutions were less likely to have academic positions than those who received $\mathrm{PhDs}$ from large majority-serving institutions ( $\mathrm{OR}=0.319, p<0.031)$. For those not in academic positions, those receiving $\mathrm{PhDs}$ from majority-serving institutions were more likely than those receiving $\mathrm{PhDs}$ from minority-serving institutions to work at large companies compared with working at nonprofits (OR $=5.524$, $p<0.05)$. As previously discussed, those who felt they were prepared for the transition from undergraduate to graduate school were more likely to currently be in academic positions than those who felt they were not prepared for this transition (OR $=2.202, p<0.005)$. Moreover, when respondents were asked to rank listed factors that we considered may have been important for their career success based on our own personal experiences (quantitative), academic URMs chose, in order of preference, 1) NIH- or NSF-funded specific programs to increase URM participation, 2) peers, 3) financial support, 4) opportunities for exploring science (e.g., conferences), and 5) mentorship as factors that played a significant role in their development as scientists. In contrast, nonacademic respondents selected 1) mentorship, 2) opportunities for exploring science, 3) financial support, 4) peers, 5) NIH- or NSF-funded specific programs to increase URM participation (Figure 3).

\section{Self-Reported Enablers of Success (Qualitative)}

We included an open-ended question about any other enablers of success not covered in the survey that participants thought important. The responses were collected and tallied to determine the most common subjective factors related to having a successful career. The most common responses, in order of preference (Table 1), were 1) mentorship or having a professional role model, 2) persistence and determination, 3) non-science support system (e.g., family and friends), 4) early undergraduate exposure to educational programs (e.g., NIH- or NSF-sponsored informative programs, conferences, higher education), 5) institutional culture of commitment for URM development, 6) financial support (e.g., NIH- or NSF-funded specific programs 


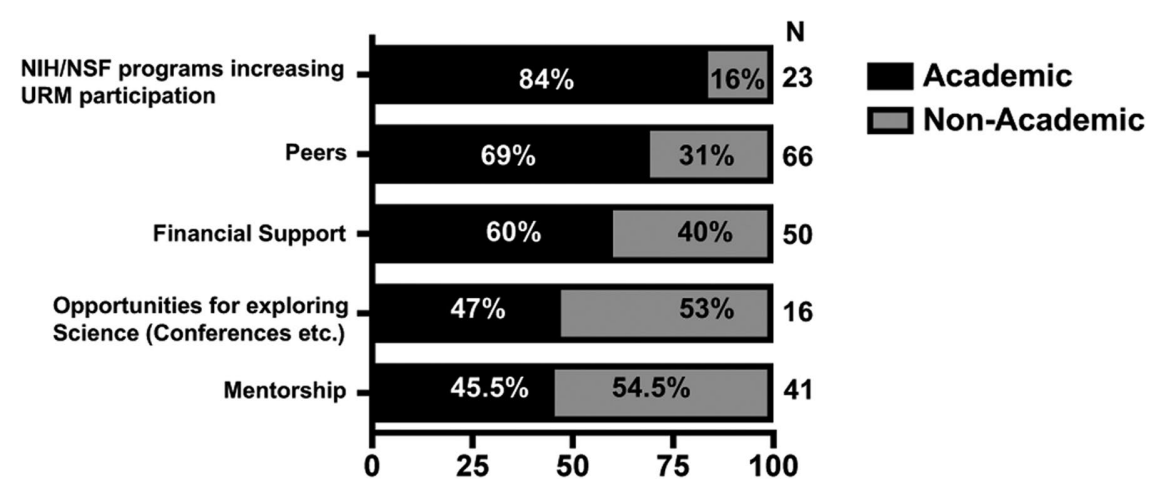

FIGURE 3. Respondents in academic and nonacademic research positions differ in factors important for their careers. Percentages in bars denote responses for the indicated factors. $N$ indicates number of responses for each factor.

in their responses (Table 2). Mentorship was the single most important self-identified factor critical to our respondents' success, especially during the later stages of their training, such as their postdoctoral years. This observation is of special interest because it suggests actionable steps that could be taken to enhance the likelihood of URMs in the research workforce, especially among nonacademic URMs or those interested in nonacademic science careers.

\section{Education and Training}

Our findings indicate that community college attendance, while proportionally low, is a larger factor for those who are recently starting their careers compared with those

to increase URM participation), and 7) personal beliefs (e.g., luck and religion). A few individuals emphasized that the lack of NIH- or NSF-funded specific programs to increase diversity in academia (e.g., postdoctoral training to faculty position), facilitated networking building, and enhanced grant-writing skills are workable factors that may contribute to improve URM success. Interestingly, one respondent highlighted the importance of stratifying U.S.-born and internationally born minority populations to understand the different needs of these individuals. Although this is a relevant issue, it is out of the scope of the current study, given that we did not ask for or collect data differentiating U.S.-born and internationally born URMs. This is a provocative area of investigation for future studies.

\section{DISCUSSION}

To our knowledge, this is the first comprehensive survey that has attempted to identify the factors that may influence the success of URM life scientists. The results could provide important information for the design and implementation of institutional and government programs aimed at promoting success with the goal of reducing disparities between the prevalence of URMs and other groups in the research workforce. For example, URMs in academic careers had opposite opinions to their nonacademic colleagues about the factors that were important for their success. Higher education institutions and U.S. government funding agencies should be aware of these differences among minority groups in the science pipeline to enhance their efforts to successfully recruit and retain faculty and researchers, as URMs have great potential to influence change in a diverse scientific workforce. Although the experiences of each of the respondents to our survey are unique, several themes emerged

TABLE 1. Most common enablers of success self-reported by URMs (in order of preference)
1. Mentorship
2. Persistence and determination
3. Strong non-science support system
4. Early exposure to scientific or educational programs
5. Institutional culture of commitment for URM development
6. Financial support
7. Personal beliefs

with established careers. In addition, participation in undergraduate research, more likely to happen at a large majority-serving institution, was associated with being prepared for the transition to graduate school and was likely to be followed by postdoctoral training in a larger ( $>16$ persons) lab. Furthermore, respondents in academic positions were more likely to feel they were prepared for the transition to graduate school. Similar interest for early research experiences in students beginning their careers in either a 2- or 4-year college has been described (Russell et al., 2007). Early hands-on research experience correlates with outcomes such as persistence in a career as a scientist, an enabler of success self-reported by the respondents (Russell et al., 2007). Many of the respondents had early exposure to research as undergraduates, a widely recognized factor that encourages students to pursue advanced degrees and careers in STEM fields. Undergraduate research opportunities help to guide students' real interest in pursuing a career in research and encourage students who are not anticipating attending graduate school to alter their professional direction to obtain their $\mathrm{PhD}$ degrees (Villarejo et al., 2008). Additionally, undergraduate research can provide URM students with an ongoing source of one-on-one mentorship that is difficult to achieve in the classroom, allowing these students to explore and discover a passion or multiple possibilities they did not know existed (Madan and Teitge, 2013).

TABLE 2. Common correlates of success for URM biomedical scientists

1. Community college attendance (for those recently starting their careers)

2. Feeling prepared for transition from undergraduate to graduate training, participation in undergraduate research, and attendance at a large majority-serving institution for undergraduate degree

3. Attendance at a large majority-serving institution for graduate degree for those in academic positions

4. Publications as a postdoc, regardless of journal IF, for those in academic positions

5. Living in a home as an undergraduate where highest degree is $\mathrm{PhD} / \mathrm{MD}$ or other professional degree, undergraduate research experience, feeling prepared for transition from undergraduate to graduate, and academic position

6. Family member not in science as role model 
Even though approximately half of the respondents (52\%) obtained their bachelor's degrees in either small majority-serving institutions or small and large minority-serving institutions, the majority of the respondents pursued their $\mathrm{PhD}$ degrees in large majority-serving institutions, and those who received PhDs at large minority-serving institutions were less likely to have academic positions than those who received $\mathrm{PhDs}$ from large majority-serving institutions. A plausible explanation for URM students' predilection to pursue graduate studies at large majority-serving institutions, and for those who received $\mathrm{PhDs}$ at large minority-serving institutions to be less likely to have academic positions, is the absence or limited research infrastructure and opportunities at small or URM institutions (Carrero-Martinez, 2011). These constraints at URM institutions may lead students to acquire hands-on research experiences through $\mathrm{NIH} / \mathrm{NSF}$ summer research opportunities or articulated agreements with large research-intensive institutions where URMs may recognize the vast number of possibilities and resources available for them to become more competitive and enhance their future career options as scientists or faculty members. This is made particularly evident by the fact that the majority of URMs in nonacademic careers obtained their PhDs in minority-serving institutions and did not pursue postdoctoral training. Our survey does not provide enough evidence to conclude that the association between $\mathrm{PhD}$ graduates from minority-serving institutions pursuing nonacademic careers are related to less research resources or networking opportunities available to graduates. Science postgraduates, especially those in the biological sciences, often see postdoctoral training as a way to continue and refine their research, learn to run a laboratory, and develop a broad, deep collaborative network (Kaplan, 2012). Many $\mathrm{PhD}$ graduates pursue postdoctoral training yet do not aspire to obtain a tenure-track position (Sauermann and Roach, 2016). Indeed, Gibbs and colleagues have suggested that women and URMs are less likely to be interested in research careers, and indeed, women and URMs who graduate from top50 institutions are less likely to be interested in academic careers (Gibbs et al., 2014). It is also conceivable that, due to economic reasons and the current length of postdoctoral training required for obtaining a faculty position, many URMs decide to find nonacademic positions in government agencies or the private sector. Additionally, there is little empirical evidence showing whether postdoctoral training benefits graduates pursuing nonacademic careers (Sauermann and Roach, 2016). Likewise, it is well known that factors that influence pursuing nonacademic positions among majority scientists include avoiding long and sometimes multiple low-wage postdoctoral positions, the rigorous and difficult tenure-track process, high-pressure lifestyle, and lower salaries in the academic setting (Sauermann and Roach, 2016).

Another important observation is that a majority of successful URMs reported training in small- to medium-sized laboratories for their graduate and postdoctoral research training. Small- to medium-sized laboratories can provide members with more access to, and interaction with, the principal investigator (PI), because individuals are not competing with many other lab mates for the PI's attention. In this type of setting, URMs may have more opportunities for individualized mentorship than in a larger laboratory, which facilitates the trainees' success in graduate or postdoctoral training and prepares them to transition to the next stage in their careers. Interactions with a mentor likely will increase the chances of URMs interested in academia to learn about funding opportunities and develop a consolidated network of peers to further advance their careers.

\section{Publication Record}

Our findings suggests that, for those who currently have academic positions, having publications during the postdoc is more important than the actual IF of the journals in which they are published. Most of our academic and nonacademic respondents published their graduate research work in low- to medium-IF journals and postdoctoral research in medium to high-IF journals. This observation contrasts with the popular notion that successfully obtaining an academic position requires publication in high-IF journals, which have been called admission into the golden club (Reich, 2013). The fact that this criterion does not appear to have been essential to many of our respondents is a welcome finding, because publication in high-IF journals is difficult and depends on project type, luck, and the interests of the mentor, and is thus largely out of the control of individual trainees. Feeling prepared during the undergraduate to graduate school transition was also associated with subsequent publication of papers in journals with higher IFs. While it is possible that people with more publications would be more confident in their ability to proceed with training or in a career, we did not ask respondents about their number of publications.

Given that publication in high-IF journals is believed to be advantageous in the current environment for a scientific career (Reich, 2013), it is possible that this finding reflected other considerations. For example, it is conceivable that laboratories that routinely publish in high-IF journals may be higher-pressure environments with less support than those which routinely publish in highly reputable, lower-IF journals. If that was the case, then the association could reflect differences in laboratory environment rather publication venue per se. It is also possible that, given the more than 2800 colleges and universities in the United States (i.e., members of the Association of American Colleges and Universities), obtaining an academic position at an academic institution is more dependent on publications per se, as compared with the IFs of journals in which publications appear, and different from what is more frequently discussed about R1 and peer institutions. Nevertheless, perhaps a more effective approach for recruitment and retention of URMs in academic positions would be to emphasize the importance of a balanced publication record consisting of quality and number of publications, particularly during the first years of their laboratory work, as success in those areas could translate into greater confidence going forward.

\section{Professional Society Membership}

Because our survey was disseminated largely via the membership of professional societies, our findings are less helpful with regard to those who are not members of professional societies. Our findings suggest that academics are more likely to be members of professional societies or to maintain their memberships once they join. It is likely that membership in professional societies is seen as more beneficial for those in academic careers compared with nonacademic careers. Interestingly, our data also suggest that respondents who did not publish as postdocs were more likely to be active members of URM committees in 
professional organizations than those who had published papers in journals with medium IFs. Further studies are needed to more comprehensively understand these relationships.

Our data also show that there is an association between membership in professional organizations and the presence of a professional mentor at the graduate, postdoctoral, and professional level. This highlights a critical role the societies can play in networking or identifying mentors and may be considered an actionable item to enhance URM success. For example, by increasing participation in professional societies at all levels, you can increase the likelihood of fostering a strong mentoring relationship.

\section{Family and Non-Science Role Models}

We found that there was a relationship between family educational attainment when respondents were undergraduates and subsequent careers. Respondents who lived in homes as undergraduates where the highest degree was $\mathrm{PhD} / \mathrm{MD}$ or other professional degree were more likely to participate in undergraduate research, feel prepared for transition from undergraduate to graduate, and end up in academic positions. Additionally, participants who did not have a role model in the family who was in science felt more prepared to transition from undergraduate to graduate school. Interestingly, persistence and determination was ranked second on the list of self-reported enablers of success, suggesting that individuals may perceive a scientific career as an opportunity to advance professionally and socially and may be more persistent and determined.

The majority of respondents reported role models as important in their careers, although most reported that this role model was a family member who was not in science. Unlike other variables, such as family education and the presence of family role models, which are essentially deterministic and cannot be altered later in life for any individual, it is possible to focus on policies that strengthen the availability of good professional mentors in the graduate and postgraduate years. For example, URMs can be steered to train with individuals with strong mentoring records, and younger faculty can be trained to become stronger mentors to URMs. Recent efforts to enhance mentoring, such as the National Research Mentoring Network (NRMN; https://nrmnet.net), whose main objective is to enhance the diversity of the NIH-funded research workforce, provides URM students, postdocs, and junior faculty with nationwide access to evidence-based mentorship and professional development in the life sciences by experienced URM and non-URM faculty, is a positive step toward this goal. Although early (Russell et al., 2007) and consistent access (Zydney et al., 2002) to research mentors and adequate resources may provide cumulative benefits (Mullen and Hutinger, 2008; Ginther et al., 2011), the mentoring needs of URMs may differ from non-URM students (Tillman, 2001; Santos and Reigadas, 2002), which suggests a need to identify the variables that lead to success in the mentormentee relationship, with particular emphasis on the URM trainees.

\section{Limitations of This Study}

Owing to the lack of data on factors contributing to the success of URM scientists in the life sciences, our study has several limitations. First, URMs and non-URMs were not compared, and this distinction may be important in identifying with certainty which success factors specifically apply to members of the minority groups. Nevertheless, we exclusively wanted to document their perspectives and experiences about their success in the life sciences that may or may not be similar in other groups. In addition, the survey was designed and discussed by URM individuals who are currently employed in academic and nonacademic positions, bringing their particular experiences to this study. Second, only members belonging to professional societies were surveyed, missing those scientists who are not affiliated with any organization. We believe that only a negligible number of URM life scientists are not currently affiliated to a professional society, making it difficult to alter the results presented and obtained by this survey. Third, we defined success as the length of a scientific career, assuming that the longer a scientist stays in a career post, the longer and greater his or her success. This assumption suggests that young faculty or newer job holders may look unsuccessful just because of the early-career status of their current positions. Finally, due to the dearth of information on factors associated with success of URM life scientists, we relied on our own experiences and those of the CMIIM members to develop a survey covering what we considered to be the most important topics for success as a life scientist.

In summary, our survey identifies important variables that contribute to keeping URMs in the leaky pipeline that constitutes the road to academic and research careers. Although personality traits such as persistence and determination were key factors identified in success, our survey provides an optimistic picture, given that the majority of the other variables identified as important can be acted upon by local and national institutions in their efforts to increase the numbers of URMs in the academic and research workforces. In this regard, the identification of the mentor-mentee relationship as the most important parameter of subsequent success for many URMs suggests the need to identify the variables of success and find mechanisms to strengthen this relationship.

\section{ACKNOWLEDGMENTS}

We are grateful to the survey participants and the minority committees of each of the professional societies who participated in the preparation and facilitated the distribution of the survey. We are also grateful for the assistance of Stephen Parry of the Cornell Statistical Consulting Unit and for anonymous comments by the reviewers that improved the paper. L.R.M. was supported by the National Institute of General Medical Sciences of the NIH under award number R15GM117501. L.R.M. has an appointment in the Infectious Diseases cluster of the Border Biomedical Research Center (National Institute on Minority Health and Health Disparities award number 2G12MD007592), UTEP's Research Centers in Minority Institutions Program. A.C. was supported by the NIH under award numbers R01HL059842, R37AI033142, and R01AI052733. A.A. was supported by the NIH under award numbers AI051626, AI065566, AI073955, AI126814, and AI129422.

\section{REFERENCES}

Allen-Ramdial S. A., \& Campbell A. G. (2014). Reimagining the pipeline: Advancing STEM diversity, persistence, and success. BioScience, 64, 612618.

Carrero-Martinez F. A. (2011). Education. Rethink summer student research Science, 334, 313 
Chemers M. M., Zurbriggen E. L., Syed M., Goza B. K., \& Bearman S. (2011). The role of efficacy and identity in science career commitment among underrepresented minority students. Journal of Social Issues, 67, 469491.

Cropsey K. L., Masho S. W., Shiang R., Sikka V., Kornstein S. G., \& Hampton C. L. (2008). Why do faculty leave? Reasons for attrition of women and minority faculty from a medical school: Four-year results. Journal of Women's Health, 17, 1111-1118.

Espinosa L., \& McDonough P. (2016, June 24). The Supreme Court frees colleges to sensibly pursue diversity. Chronicle of Higher Education. Retrieved July 6, 2016, from www.chronicle.com/article/The-Supreme -Court-Frees/236921

Fang D., Moy E., Colburn L., \& Hurley J. (2000). Racial and ethnic disparities in faculty promotion in academic medicine. Journal of the American Medical Association, 284, 1085-1092.

Gibbs K. D., Basson J., Xierali I. M., \& Broniatowski D. A. (2016). Decoupling of the minority $\mathrm{PhD}$ talent pool and assistant professor hiring in medical school basic science departments in the US. eLife, 5, e21393.

Gibbs K. D. Jr., McGready J., Bennett J. C., \& Griffin K. (2014). Biomedical science Ph.D. career interest patterns by race/ethnicity and gender. PLoS One, 9, e114736.

Ginther D. K., Schaffer W. T., Schnell J., Masimore B., Liu F., Haak L. L., \& Kington R. (2011). Race, ethnicity, and NIH research awards. Science, 333 1015-1019.

Griffin K., Gibbs J., Kenneth D., Bennett J., Staples C., \& Robinson T. (2015). "Respect me for my science": A Bourdieuian analysis of women scientists' interactions with faculty and socialization into science. Journal of Women and Minorities in Science and Engineering, 21, 159-179.

Hassouneh D., Lutz K. F., Beckett A. K., Junkins E. P., \& Horton L. L. (2014). The experiences of underrepresented minority faculty in schools of medicine. Medical Education Online, 19, 24768.

Kaplan K. (2012). Postdoc or not? Nature, 483, 499-500.

Leboy P. S., \& Madden J. F. (2012). Limitations on diversity in basic science departments. DNA and Cell Biology, 31, 1365-1371.

Madan C. R., \& Teitge B. D. (2013, May 1). The benefits of undergraduate research: The student's perspective. The Mentor: An Academic Advising Journal. Retrieved June 27, 2016, from https://dus.psu.edu/mentor/ 2013/05/undergraduate-research-students-perspective

Mahoney M. R., Wilson E., Odom K. L., Flowers L., \& Adler S. R. (2008). Minority faculty voices on diversity in academic medicine: Perspectives from one school. Academic Medicine, 83, 781-786.

McMurtrie B. (2016, May 25). A "devastating account" of diversity at Yale. Chronicle of Higher Education. Retrieved June 12, 2016, from www .chronicle.com/article/A-Devastating-Account-of/236598]

Mullen C. A., \& Hutinger J. L. (2008). At the tipping point? Role of formal faculty mentoring in changing university research cultures. Journal of In-Service Education, 34, 181-204.

Palepu A., Carr P. L., Friedman R. H., Ash A. S., \& Moskowitz M. A. (2000). Specialty choices, compensation, and career satisfaction of underrepresented minority faculty in academic medicine. Academic Medicine, 75, 157-160

Peralta E. (2015, November 9). Amid controversy, University of Missouri president and chancellor step down. National Public Radio. Retrieved December 15, 2015, from www.npr.org/sections/thetwo-way/ 2015/11/09/455324174/amid-controversy-university-of-missouri -system-president-resigns

Pololi L., Cooper L. A., \& Carr P. (2010). Race, disadvantage and faculty experiences in academic medicine. Journal of General Internal Medicine, 25, 1363-1369.

Price E. G., Gozu A., Kern D. E., Powe N. R., Wand G. S., Golden S., \& Cooper L. A. (2005). The role of cultural diversity climate in recruitment, promotion, and retention of faculty in academic medicine. Journal of General Internal Medicine, 20, 565-571.

Reich E. S. (2013). Science publishing: The golden club. Nature, 502, 291-293

Rodriguez C., Kirshstein R., Banks-Amos L., Jones W., Espinosa L., \& Watnick D. (2012). Broadening participation in STEM: A call to action. Washington, DC: American Institutes for Research.

Rodriguez J. E., Campbell K. M., Fogarty J. P., \& Williams R. L. (2014). Underrepresented minority faculty in academic medicine: A systematic review of URM faculty development. Family Medicine, 46, 100-104.

Russell S. H., Hancock M. P., \& McCullough J. (2007). The pipeline. Benefits of undergraduate research experiences. Science, 316, 548-549.

Santos S. J., \& Reigadas E. T. (2002). Latinos in higher education: An evaluation of a university faculty mentoring program. Journal of Hispanic Higher Education, 1, 40-50.

Sauermann H., \& Roach M. (2016). Why pursue the postdoc path? Science, $352,663-664$

Tillman L. C. (2001). Mentoring African American faculty in predominantly white institutions. Research in Higher Education, 42, 295-325.

Villarejo M., Barlow A. E., Kogan D., Veazey B. D., \& Sweeney J. K. (2008) Encouraging minority undergraduates to choose science careers: Career paths survey results. CBE-Life Sciences Education, 7, 394-409.

Whittaker J. A., \& Montgomery B. L. (2012). Cultivating diversity and competency in STEM: Challenges and remedies for removing virtual barriers to constructing diverse higher education communities of success. Journal of Undergraduate Neuroscience Education, 11, A44-A51.

Whittaker J. A., Montgomery B. L., \& Martinez Acosta V. G. (2015). Retention of underrepresented minority faculty: Strategic initiatives for institutional value proposition based on perspectives from a range of academic institutions. Journal of Undergraduate Neuroscience Education, 13, A136145.

Zambrana R. E., Ray R., Espino M. M., Castro C., Cohen B. D., \& Eliason J. (2015). "Don't leave us behind": The importance of mentoring for underrepresented minority faculty. American Educational Research Journal, $52,40-72$

Zydney A. L., Bennett J. S., Shahid A., \& Bauer K. W. (2002). Impact of undergraduate research experience in engineering. Journal of Engineering Education, 91, 151-157. 